\title{
Anisotropy in Oxidation of Zirconium Surfaces from Density \\ Functional Theory Calculations
}

\author{
Tsu-Wu Chiang (蔣祖武), Aleksandr Chernatynskiy, Mark J. Noordhoek, Susan B. Sinnott \\ and Simon R. Phillpot* \\ Department of Materials Science and Engineering \\ University of Florida \\ Gainesville, FL 32611, USA
}

To be submitted to Computational Materials Science

*Corresponding author: sphil@mse.ufl.edu 


\begin{abstract}
This work uses density functional theory calculations to analyze the energy barriers for oxygen migration into the basal and prismatic surfaces of zirconium. Specifically, the migration energy barriers between each octahedral site and tetrahedral site in the basal surface, prism surface, and the bulk are determined. The possible oxygen migration paths in each system are also analyzed. Oxygen has higher energy barriers to penetrating the basal surface than the prism surface. It also has a lower energy barrier to escape from basal surface than from the prism surface. This is consistent with the experimental observation that the prism plane of zirconium oxidizes more quickly than the basal plane.
\end{abstract}




\section{Introduction}

Zirconium (Zr) based alloys are widely used as the clad for nuclear fuel because of their structural stability and low thermal neutron absorption cross-section [1]. The prototypical clad in boiling water reactors and pressurized water reactors is Zircaloy 2, which is more than $98 \% \mathrm{Zr}$, with $1.5 \% \mathrm{Sn}, 0.12 \% \mathrm{Fe}, 0.1 \% \mathrm{Cr}, 0.1 \% \mathrm{Ni}$ and other components [2]. More recent clads, such as Zircaloy 4, Zirlo ${ }^{\mathrm{TM}}$ or $\mathrm{M} 5^{\circledR}$ [3], contain slightly different compositions than Zircaloy 2 and are engineered for optimal performance in a number areas, including oxidation .

The oxidation of $Z r$ based alloys has been widely investigated because a thin film of zirconium oxide has a dense structure that can inhibit fission gas penetration through the metal. As long ago as 1970, a study by Fehlner and Mott [4] of the transition from chemisorption to 3-D oxide and anion migration during oxide growth showed that at low temperature $(\sim 300 \mathrm{~K})$ the oxidation process depends on the oxygen partial pressure. The metal crystallographic orientation could not influence the oxidation rate. In a study of anodic oxidation, Davies et al. found that Zr oxidation behavior only involves the oxygen migration rather than Zr migration [5]. It has also been determined that the presence of oxygen in Zr subsurface interstitial sites can prevent the further penetration of oxygen, leading to a decreased diffusion rate $[6,7]$.

There have been a number of experimental studies of oxygen absorption on the basal (0001) Zr surface. A study by Wang et al. [8] showed that at 0.5 monolayer (ML) coverage, half of the $\mathrm{O}$ atoms (i.e., $0.25 \mathrm{ML}$ ) occupy the octahedral sites between the first and second layers of the $\mathrm{Zr}$ subsurface, while the other half occupy octahedral sites between the second and third layers. They also showed that once the oxygen coverage 
reaches $2 \mathrm{ML}$, the oxygen ions reside in the surface face centered cubic (SFCC) sites and in the tetrahedral sites between first and second subsurface layers [9]. Other studies characterized the oxidation rate using electron microscopy (TEM and SEM) [10, 11]. These studies found that the prismatic $\{10 \overline{1} 0\}$ surface has a much faster oxidation rate than the basal (0001) surface.

Studies using density functional theory (DFT) calculations with the local-density approximation (LDA) approximation have also analyzed oxygen absorption by the $\mathrm{Zr}$ basal surface [12-14]. These concluded that the energetically favored oxygen absorption sites are the octahedral sites between the second and third layers [12]. However, more recent calculations with the generalized gradient approximation (GGA) using the double oxygen layer model, in which oxygen is placed in two layers, arrived at a different conclusion: the energetically favored sites are the SFCC sites [13], as seen in Fig. 1. Another GGA study using a multiple-layer adsorption model (MLAM), with oxygen in multiple layers, indicated that at $0.25 \mathrm{ML}$ the SFCC site is energetically favored. In this model, as the coverage increases to $0.5 \mathrm{ML}$ a combination of SFCC sites and octahedral sites is the most stable, as indicated in Fig. 1 [14]. These GGA calculations $[13,14]$ also suggest that while thermal diffusion allows oxygen to cross the energy barrier from the SFCC sites to the subsurface, the occupation of subsurface sites can prevent further oxygen penetration [13]. A recent LDA calculation determined [15] that strain on the surface can change the most stable sites on $\mathrm{Zr}$ basal surface from SFCC sites to octahedral sites.

Little attention has focused on analyzing the energy barriers for oxygen penetration into the $\mathrm{Zr}$ basal and prism surfaces. These energy barriers are important 
because they can be expected to play an important role in oxidation. While one DFT study found that oxygen has a lower energy barrier for migration between SFCC sites than from SFCC sites to subsurface octahedral interstitial sites, it did not compare the basal and prism surfaces [16]. Hence, with the objective of understanding the large difference in their oxidation rates, in this work we determine the paths and energy barriers associated with the oxygen migration into $\mathrm{Zr}$ basal and prism surfaces.

\section{Methods and Bulk Migration Barriers}

The DFT calculations are performed using the Vienna ab initio Simulation Package (VASP) [17, 18] with the projector augmented-wave (PAW) method [19].The GGA approximation is chosen since it performed well in the study of the multiple-layer adsorption model [14]. The Perdew-Burke-Ernzerhof function (PBE) [20] is used to describe the gradient of the electron density. The kinetic energy cutoff is $400 \mathrm{eV}$, which is the same as used in an earlier DFT study of $\operatorname{Zr}$ [14]. An $8 \times 8 \times 8 \mathrm{k}$-point mesh is used for the bulk supercell. HCP-structured single crystal Zr structure yields a lattice constant of $\mathrm{a}=0.324 \mathrm{~nm}, \mathrm{c}=0.515 \mathrm{~nm}$, which gives $\mathrm{c} / \mathrm{a}=1.595$. These calculated values are consistent with the experiment values of $\mathrm{a}=0.323 \mathrm{~nm}, \mathrm{c}=0.515 \mathrm{~nm}$ and $\mathrm{c} / \mathrm{a}=1.593$, and in good agreement with previous DFT results [14, 21]. Previous experimental work showed that the $Z r$ thermal expansion does not significantly affect the c/a ratio. At 950 $\mathrm{K}$, the experimental c/a is measured to be 1.597 [22], which is only $0.25 \%$ different from the calculated $0 \mathrm{~K}$ value. Moreover, the volume expansion at $950 \mathrm{~K}$ is also less than $1 \%$ [22]. Thus, the structure found in $\mathrm{T}=0 \mathrm{~K}$ DFT calculations can reasonably be compared to previous experimental studies $[8,10]$. 
The climbing image nudged elastic band (cNEB) method [23, 24] is used to calculate the atom migration saddle points and the migration energy barriers. In each migration path considered here, six images are used. Before structural equilibration, the interval of oxygen between each image along the migration path is about $1 \AA$ or less. Our results show that this resolution is enough to reliably determine the height of the energy barriers.

To put the surface effects into context, it is first necessary to understand migration within the bulk. There are two distinct interstitial sites: an octahedral $(O)$ site and a tetrahedral (T) site. While an oxygen in an $\mathrm{O}$ site lies half way between two $\mathrm{Zr}$ planes and has six $\mathrm{Zr}$ nearest neighbors, an oxygen in a $\mathrm{T}$ site lies closer to one $\mathrm{Zr}$ layer than the other and has only three $\mathrm{Zr}$ nearest neighbors. As a result the energy of the oxygen is higher energy in the $T$ sites than in the $O$ sites, and is thus less strongly bound to the T sites. The DFT calculations confirm this and are consistent with the results of previous studies $[12,14]$.

The possible near-neighbor migration jumps among interstitial sites are illustrated in Fig. 1c, for which we determine all of the energy barriers. Jumps shown as dotted lines in Fig. 1c have high energies and are not part of the lowest energy path; jumps shown as solid lines are part of the lowest energy paths. For example, the barriers for migration from an $\mathrm{O}$ site through a $\mathrm{Zr}$ plane to either an $\mathrm{O}$ site or a $\mathrm{T}$ on the other side (interlayer migration) are extremely high because during such a jump the atoms approach a $\mathrm{Zr}$ atom pushing it off its lattice site. The lowest energy path for interlayer migration is $\frac{1}{6}[0001]$ from $\mathrm{T}$ to $\mathrm{T}$ with a barrier height of only $0.1 \mathrm{eV}$. For migration among sites lying between the same two $\mathrm{Zr}$ layer (intralayer migration), the barriers for 
$\mathrm{O}$ to $\mathrm{O}$ and $\mathrm{T}$ to $\mathrm{T}$ paths are high, while the lowest is that between $\mathrm{O}$ and $\mathrm{T}(1.72 \mathrm{eV})$ and between $\mathrm{T}$ and $\mathrm{O}(0.83 \mathrm{eV})$. Each $\mathrm{O}$ site has 6 equivalent intralayer neighbor $\mathrm{T}$ sites, three above it and three below it, lying along $\frac{1}{12}<40 \overline{4} 1>$, while each T site has 3 equivalent $\mathrm{O}$ neighbor sites, all of which are either above it or below it. As a result the paths for long-range migration in any crystallographic direction are made up of combinations of three distinct jumps: low energy interlayer $\mathrm{T}$ to $\mathrm{T}$ jumps, and higher energy intralayer $\mathrm{O}$ to $\mathrm{T}$ and $\mathrm{T}$ to $\mathrm{O}$ jumps. In particular, the net path for migration along [0001] involves three distinct jumps: $\mathrm{O}$ to $\mathrm{T}, \mathrm{T}$ to $\mathrm{T}$ and to $\mathrm{T}$ to $\mathrm{O}$. Migration within the basal plane only requires $\mathrm{O}$ to $\mathrm{T}$ and $\mathrm{T}$ to $\mathrm{O}$ jumps. However, $\mathrm{In}$ this case there may also be multiple interlayer ( $T$ to $T$ ) jumps as the energy barrier to these is so low. Thus the migration path in any direction is dominated by the highest energy $\mathrm{O}$ to $\mathrm{T}$ jump, which is required for diffusion both parallel to and perpendicular to the c-axis. This result is consistent with previous experimental study of oxygen diffusion in $\alpha-Z r$ which showed that an anisotropy of bulk diffusion in $\alpha-Z r$ is small [25].

\section{Migration barriers into (0001) and $\{10 \overline{1} 0\}$ surfaces}

We now turn to determining the migration barriers in the surface region. To perform the calculations of oxygen migration, HCP basal (0001) and prism $\{10 \overline{1} 0\}$ surfaces are created. Schematics of these two surfaces are given in Fig. 2. The planar density of the basal plane is $1.1 \times 10^{13}$ atoms $/ \mathrm{mm}^{2}$ which is higher than the density of the prism plane: $6.0 \times 10^{12}$ atoms $/ \mathrm{mm}^{2}$. The supercell for the surface calculations has eight metal layers with $2 \times 2$ surface unit cells, as shown in Figs. 3 and 4 . A vacuum region of the same thickness as the thickness of the metal film is added and an $8 \times 8 \times 1$ 
k-point mesh is used in the calculations, which is similar to that used in a previous study [14]. Throughout these calculations, atoms in the bottom four atomic layers are fixed to mimic the effects of an infinitely thick system.

In Figs. 2 and 3 , the $\mathrm{O}$ and $\mathrm{T}$ sites are numbered to denote their positions relative to the surface (layer 1). For the (0001) basal surface, Fig. 2, the $O(12)$ site is an octahedral site between layers 1 and 2, while $O(01)$ lies above the $Z r$ surface; this $O(01)$ site is the SFCC. The $T(12 A)$ site lies closer to layer 1, while the $T(12 B)$ lies closer to layer 2. In Fig. 3 of the prism surface, the $O$ and $T$ sites are denoted as in the basal surface and thus do not relate directly to the layer number.

Table 1 gives the energy barriers for migration into and out of the (0001) and $\{10 \overline{1} 0\}$ surfaces. For both surfaces, the lowest energy path is essentially the same as the bulk migration path. However, the energy barriers are modified.

Figure 4 illustrates the lowest energy path for migration into and out of the (0001) surface. The overall lowest barrier path is from $\mathrm{O}(01)$ to $\mathrm{O}(12)$ to $\mathrm{T}(12 \mathrm{~B})$ to $\mathrm{T}(23 \mathrm{~A})$ to $O(23)$ to $T(23 B)$ to $T(34 A)$ to $O(34)$. This energy barrier from $O(01)$ to $O(12)$ is smaller than for migration between equivalent $O$ sites in the bulk because an oxygen sitting on the surface only has half the number of $Z r$ bonds as the corresponding interstitial in the $\mathrm{Zr}$ structure. Upon the oxygen atom movement from $\mathrm{O}(01)$ to $\mathrm{O}(12)$ site, only three O$\mathrm{Zr}$ bonds are broken, fewer than six bonds broken in the corresponding transition in the bulk. The oxygen doesn't migrate through from $\mathrm{O}(01)$ to $\mathrm{T}(01)$ to $\mathrm{T}(12 \mathrm{~A})$ to $\mathrm{O}(12)$ because the oxygen in $\mathrm{T}(12 \mathrm{~A})$ is very unstable, as a previous study indicated [13]. In our calculations, we find an oxygen in $T(12 A)$ spontaneously moves to $T(01)$ with no measurable energy barrier. The oxygen also cannot migrate directly from $\mathrm{O}(01)$ to 
$T(12 A)$ or from $T(01)$ to $O(12)$ because, as discussed in the last section in the context of bulk diffusion, the oxygen cannot cross the $\mathrm{Zr}$ layer from $\mathrm{O}$ to $\mathrm{T}$. Hence, oxygen must directly migrate from $\mathrm{O}(01)$ to $\mathrm{O}(12)$. In addition, oxygen cannot migrate directly from $T(12 A)$ to $T(12 B)$ or other equivalent sites because along these paths, the oxygen would also approach $\mathrm{Zr}$ atoms too closely.

The energy barrier for $\mathrm{T}(12 \mathrm{~B})$ to $\mathrm{T}(23 \mathrm{~A})$ is much lower than for $\mathrm{O}(01)$ to $\mathrm{O}(12)$ or for $\mathrm{O}(12)$ to $\mathrm{T}(12 \mathrm{~B})$, consistent with the behavior in the bulk. All energy barriers for transitions from $\mathrm{O}(23)$ or sites deeper in to the surface are very similar to the bulk values. This is expected because previous work showed that the energy of oxygen interstitials in these sites have close values [13]. Since the energy change in these sites are small, we could consider oxygen behavior in these sites are close to oxygen in bulk sites. Thus the surface only influences oxygen migration barrier in top two to three layers.

The oxygen migration in the $\{10 \overline{1} 0\}$ prism surface can be analyzed in a manner similar to oxygen migration in the basal surface; a sketch of the oxygen interstitial in the prism surface is shown in Fig. 3. Again the energy barriers for migration from $T$ to $T$ and from $\mathrm{O}$ to $\mathrm{O}$ are high. If the oxygen migrates through these paths, it pushes the $\mathrm{Zr}$ atoms off their lattice sites, which is energetically expensive. Thus oxygen can only diffuse along the $\mathrm{O}$ to $\mathrm{T}$ to $\mathrm{O}$ path, as in the bulk. Figure 5 shows the barriers for the lowest energy path for migration into the $\{10 \overline{1} 0\}$ surface.

The energy barrier from $\mathrm{T} 1$ to $\mathrm{O} 1$ is smaller than from $\mathrm{T}$ to $\mathrm{O}$ energy in bulk in a manner similar to that for the (0001) surface. The energy barrier from 01 to T2 is higher 
than in bulk, which is also similar to the $\mathrm{O}(12)$ to $\mathrm{T}(12 \mathrm{~B})$ barrier. The migration barriers from T2 and from sites deeper in the surface are very similar to the bulk values.

\section{Discussion}

The most important difference between the two surfaces is that the barrier from the basal surface to the first subsurface layer is substantially higher, $1.89 \mathrm{eV}$, compared to $1.22 \mathrm{eV}$ for the prism surface. In addition, the barrier for oxygen to migrate back out from the first subsurface layer onto the surface is lower for the in basal surface, $1.1 \mathrm{eV}$, than for the prism surface, $1.29 \mathrm{eV}$. High penetration barrier for the basal plane implies that this plane is more resistant to oxidation than prism plane. Difference in planar atomic density between these two surfaces was implicated in different oxidation resistance [10]. Our results, however, indicate more nuanced view of the effect. According to our calculations, the main reason for the higher penetration barrier on the basal surface is relatively large adsorption energy of the oxygen atom to the basal plane. It is found to be $0.28 \mathrm{eV}$ greater than that to the prism plane. This stronger bond is in turn a consequence of the higher atomic density in the basal plane.

The effect that these different energy barriers could have on the oxidation rate can be qualitatively understood simply by looking at associated oxygen jumping rates: $\mathrm{T}=\mathrm{v} e^{-E_{m} / K_{B} T}$

where $T$ is the jump rate, $V$ is the vibrational frequency of the atom (oxygen), $E_{m}$ is the energy barrier for migration, and $\mathrm{K}_{B}$ is the Boltzmann constant. Based on these energy barriers, and assuming that the vibrational frequencies are the same, the jump rate of oxygen into the prism surface should be $\sim 10^{11}$ higher than into the basal plane. Similarly, the migration rate from the first subsurface layer back to the surface should be 
$\sim 10^{3}$ higher for the basal surface than prism surface. Both of these processes should thus lead to a significantly lower oxidation rate for the basal surface than for the prism surface, consistent with experiment $[10,11,26]$.

\section{Conclusions}

These results demonstrate that the barriers for oxygen penetration in the basal surface and prism surface show similar trends, in that barrier for penetration from above the surface into the first subsurface layer is lower than the bulk value, while the barrier from the first subsurface layer to the second is higher. In both cases, the barriers further in the surface have rapidly approached the bulk values.

While the above analysis points to the anisotropy in the energy barriers at the surface as at least in part accounting for the strong differences in oxidation rates, they are likely not the entire story. In particular, this analysis does not take into account any microstructural elements such as grain boundaries and dislocations which can be expected to have a significant effect on diffusion.

\section{Acknowledgments}

This work was supported by DOE NEUP 12-4728. SBS acknowledges the support of the National Science Foundation (DMR-1207293). 


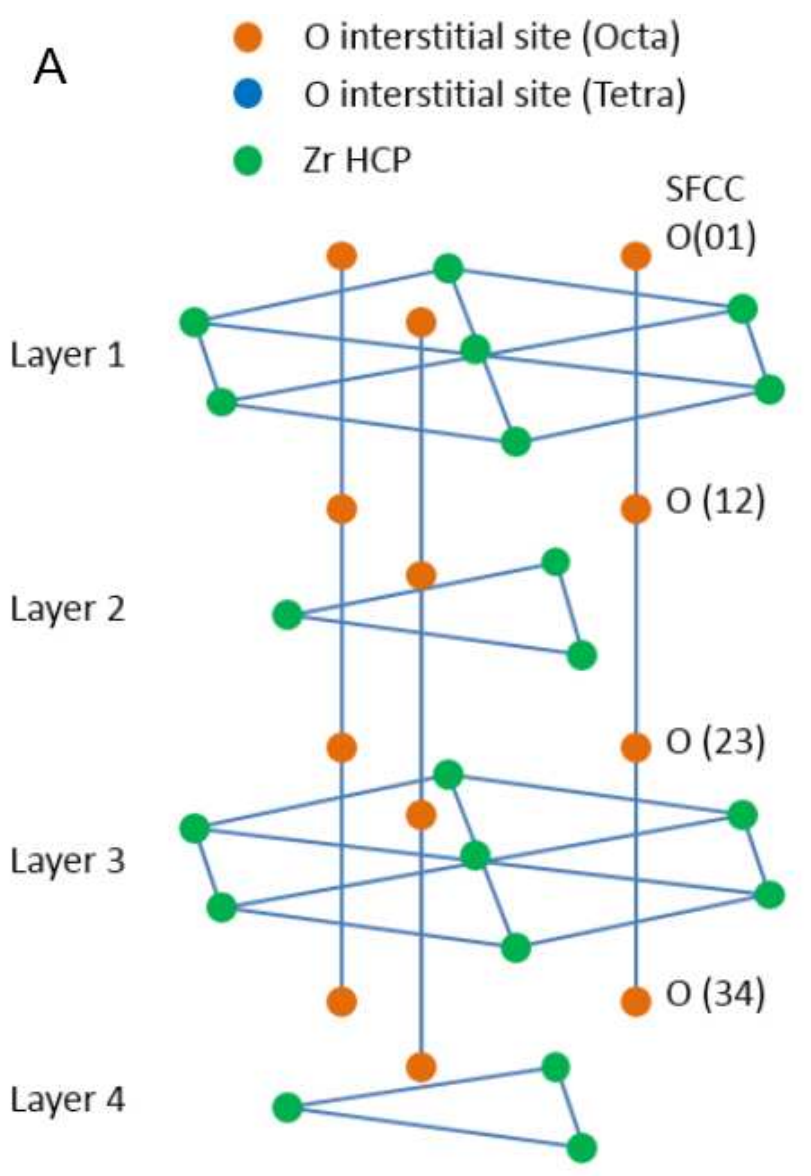

B

C

Layer 1

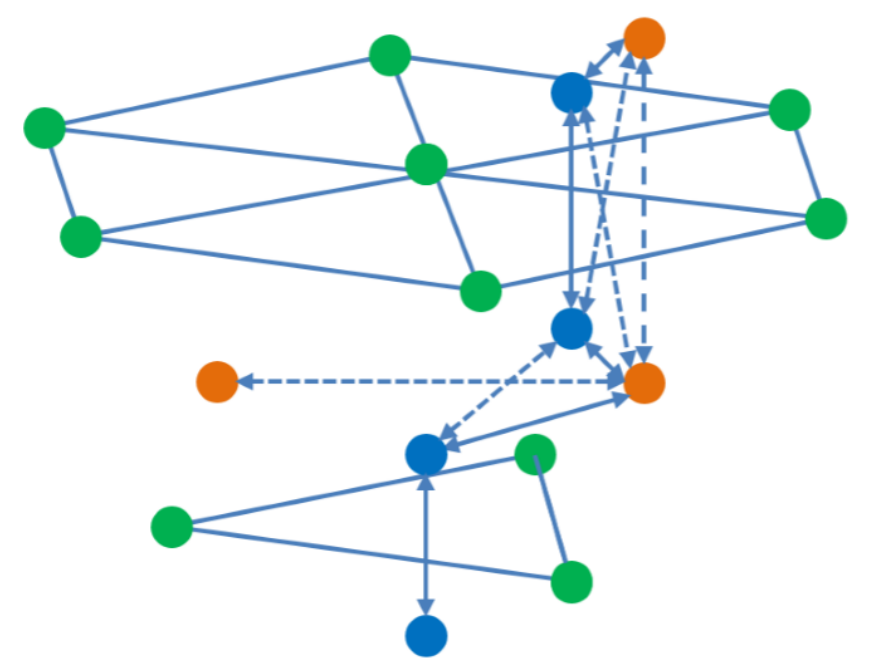

Layer 2 


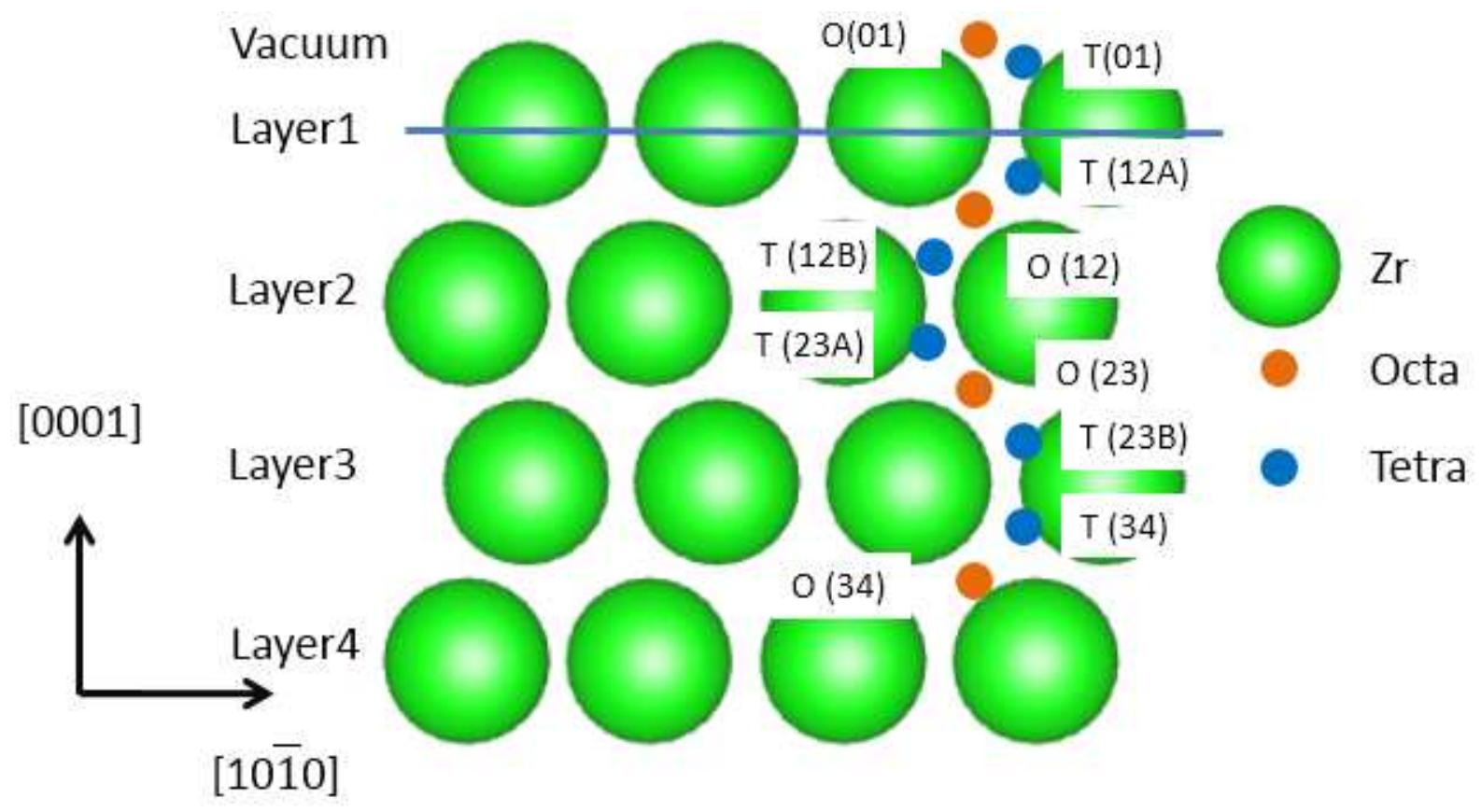

Fig. 2. Oxygen interstitial sites on (0001) basal surface. 


\section{A Vacuum}
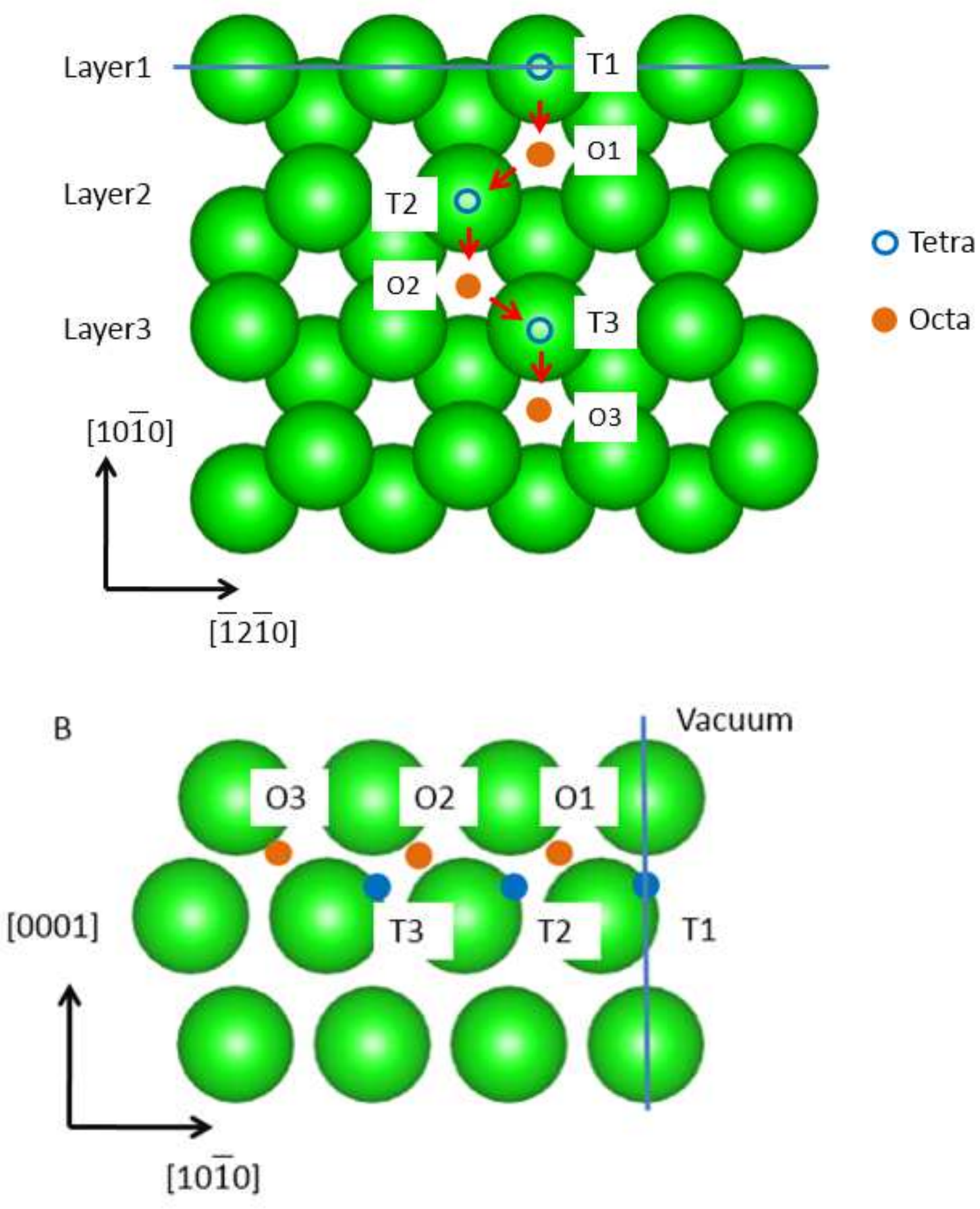

Fig. 3. Oxygen interstitial sites on the [1010] prism surface. The open circle indicates tetrahedral sites that lie directly behind the $\mathrm{Zr}$ atoms. Arrows indicate a possible oxygen penetration direction. The $A$ and $B$ are from different view to the prism surface. 


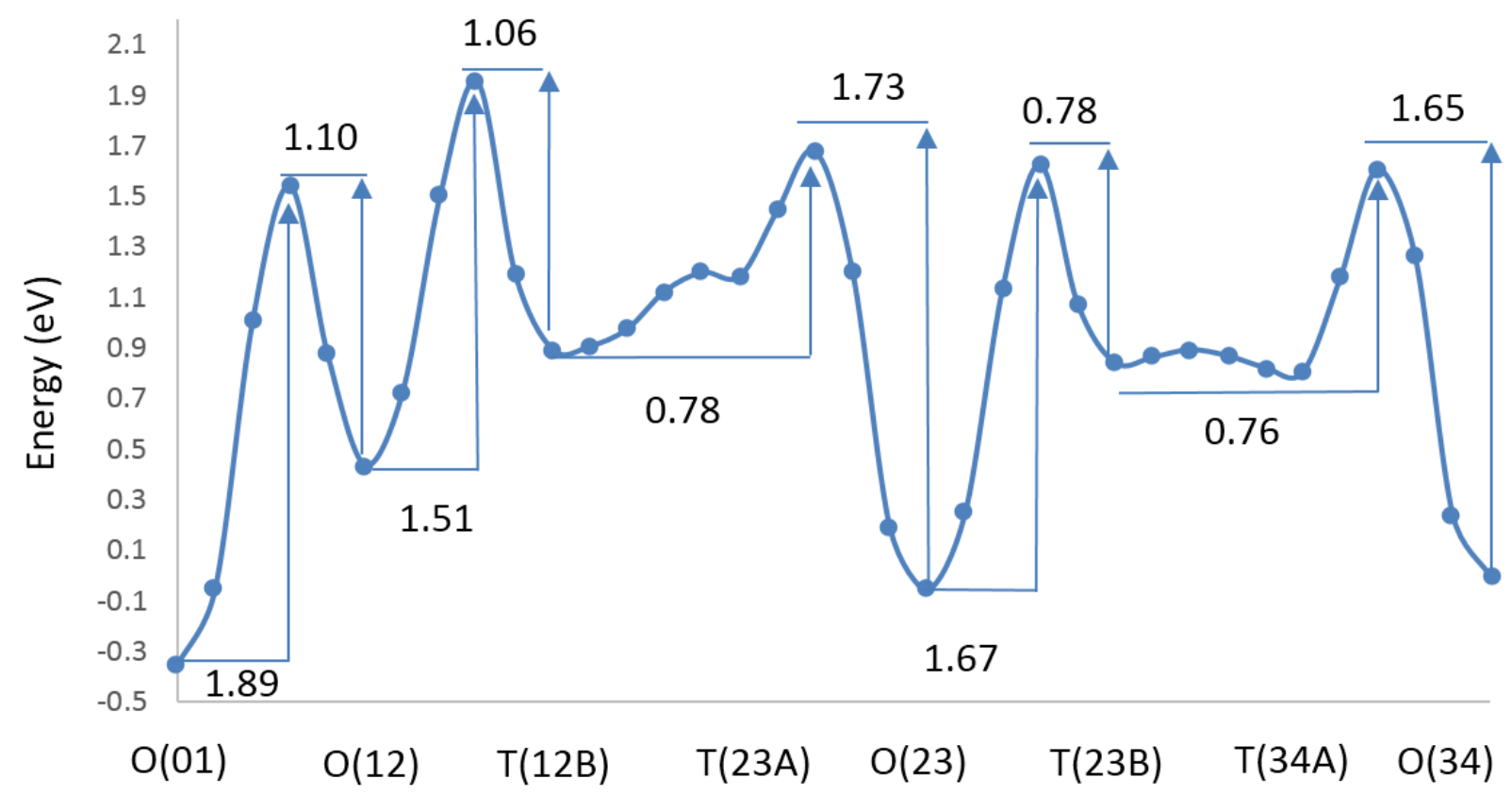

Fig. 4. The lowest energy path for oxygen migration into the basal (0001) surface of $Z$ r. The reference state is the energy of oxygen in a bulk $\mathrm{O}$ site. 


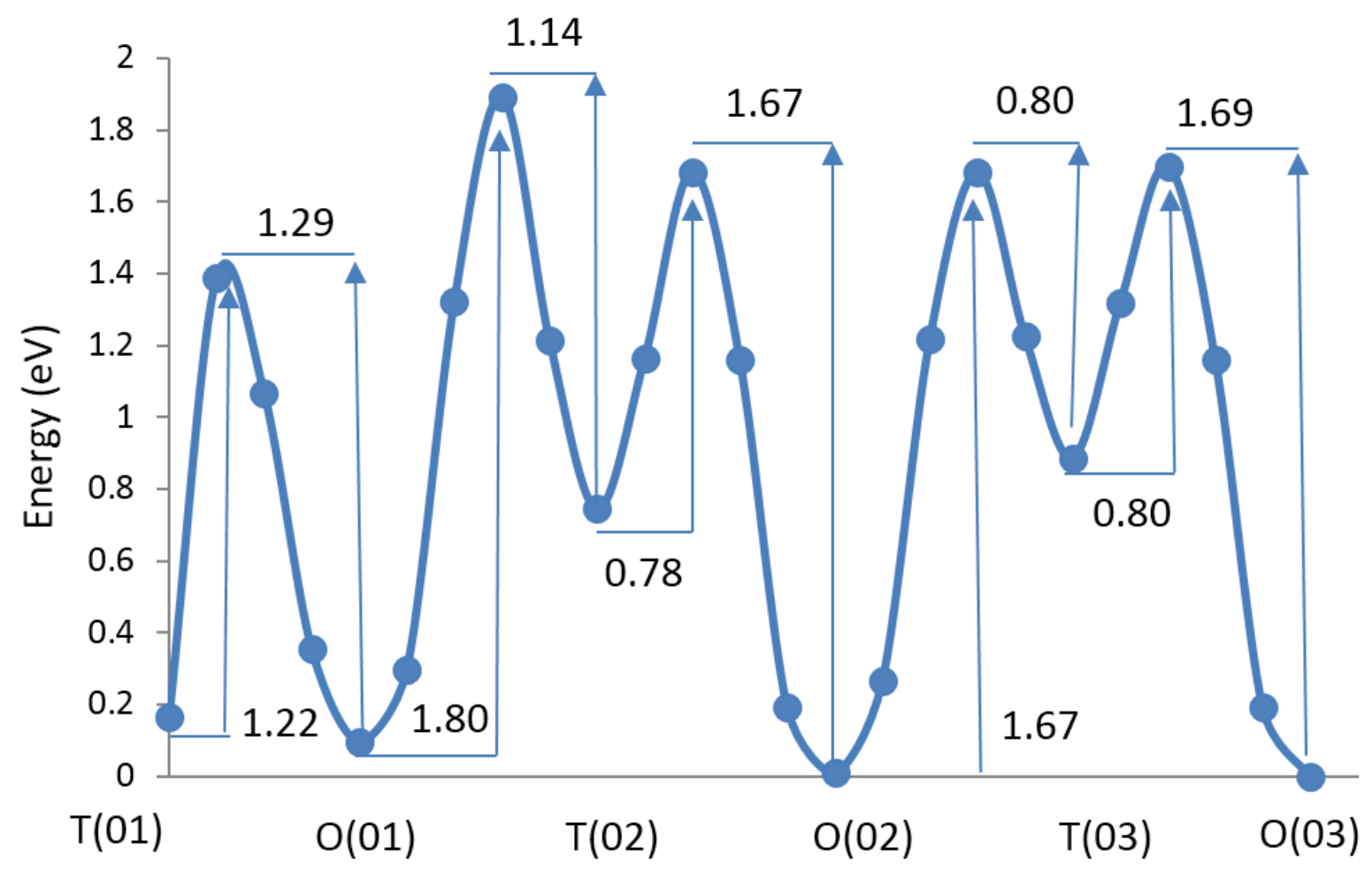

Fig. 5. The lowest energy path for oxygen migration into the prism $<10 \overline{1} 0>$ surfaces of $Z r$. The zero of energy is for an oxygen in a bulk $O$ site. 


\begin{tabular}{|c|c|c|c|}
\hline Start & End & Forward Barrier (eV) & Reverse Barrier (eV) \\
\hline \multicolumn{4}{|l|}{ Bulk } \\
\hline \multicolumn{4}{|l|}{ Interlayer } \\
\hline $\mathrm{O}$ & $\mathrm{O}$ & 2.87 & 2.87 \\
\hline $\mathrm{T}$ & $\mathrm{T}$ & 0.10 & 0.10 \\
\hline \multicolumn{4}{|l|}{ Intralayer } \\
\hline $\mathrm{O}$ & $\mathrm{T}$ & 1.72 & 0.83 \\
\hline \multicolumn{4}{|l|}{ Basal Surface } \\
\hline \multicolumn{4}{|l|}{$\mathrm{O} \rightarrow \mathrm{O}$} \\
\hline $\mathrm{O}(01)$ & $\mathrm{O}(12)$ & 1.89 & 1.10 \\
\hline $\mathrm{O}(12)$ & $\mathrm{O}(23)$ & 2.41 & 2.90 \\
\hline $\mathrm{O}(23)$ & $\mathrm{O}(34)$ & 2.81 & 2.85 \\
\hline \multicolumn{4}{|l|}{$\mathrm{T} \rightarrow \mathrm{T}$} \\
\hline $\mathrm{T}(01)$ & $\mathrm{T}(12 \mathrm{~A})$ & 0.99 & 0.00 \\
\hline $\mathrm{T}(12 \mathrm{~B})$ & $\mathrm{T}(23 \mathrm{~A})$ & 0.30 & 0.04 \\
\hline $\mathrm{T}(23 \mathrm{~B})$ & $\mathrm{T}(34 \mathrm{~A})$ & 0.05 & 0.08 \\
\hline \multicolumn{4}{|c|}{$\mathrm{O} \rightarrow \mathrm{T} \rightarrow \mathrm{T} \rightarrow \mathrm{O}$} \\
\hline $\mathrm{O}(01)$ & $\mathrm{O}(12)$ & 1.89 & 1.10 \\
\hline $\mathrm{O}(12)$ & $\mathrm{T}(12 \mathrm{~B})$ & 1.51 & 1.06 \\
\hline $\mathrm{T}(12 \mathrm{~B})$ & $\mathrm{T}(23 \mathrm{~A})$ & 0.30 & 0.04 \\
\hline $\mathrm{T}(23 \mathrm{~A})$ & $\mathrm{O}(23)$ & 0.48 & 1.73 \\
\hline $\mathrm{O}(23)$ & $\mathrm{T}(23 \mathrm{~B})$ & 1.67 & 0.78 \\
\hline
\end{tabular}

\section{Prism surface}

$\mathrm{O} \rightarrow \mathrm{T} \rightarrow \mathrm{O}$

$\mathrm{T}(01)$

$\mathrm{O}(01)$

1.22

1.29

$\mathrm{O}(01)$

$\mathrm{T}(02)$

1.80

1.14

$\mathrm{T}(02)$

$\mathrm{O}(02)$

0.78

1.67

$\mathrm{O}(02)$

$\mathrm{T}(03)$

1.67

0.80

$\mathrm{T}(03)$

$\mathrm{O}(03)$

0.80

1.69

Table. 1. Energy barriers for oxygen diffusion in Zr. Barriers are not shown for the interlayer $\mathrm{O}$ to $\mathrm{T}$ jump, the $\mathrm{O}$ to $\mathrm{O}$ and $\mathrm{T}$ to $\mathrm{T}$ intralayer jumps, or their equivalents in surfaces as they cannot take place. 


\section{REFERENCES}

[1] N. Stojilovic, E.T. Bender, R.D. Ramsier, Pro. Surf. Sci, 78 (2005) 101-184.

[2] A.J. Rothman, Potential corrosion and degradation mechanisms of Zircaloy cladding on spent nuclear fuel in a tuff repository, in, Lawrence Livermore National Laboratory

Report UCID-20172, 1984.

[3] M. Steinbrück, M. Böttcher, J. Nucl. Mater., 414 (2011) 276-285.

[4] F.P. Fehlner, N.F. Mott, Oxid. Met., 2 (1970) 59.

[5] J.A. Davies, B. Domeij, J.P.S. Pringle, F. Brown, J. Electrochem. Soc., 112 (1965) 675.

[6] K. Griffiths, J. Vac, Sci. Technol. , A6 (1988) 210.

[7] C.-S. Zhang, B.J. Flinn, P.R. Norton, Surf. Sci, 264 (1992) 1-9.

[8] Y.M. Wang, Y.S. Li, K.A.R. Mitchell, Surf. Sci, 342 (1995) 272-280.

[9] Y.M. Wang, Y.S. Li, K.A.R. Mitchell, Surf. Sci, 380 (1997) 540-547.

[10] H.G. Kima, T.H. Kima, Y.H. Jeong, J. Nucl. Mater, 306 (2002) 44-53.

[11] R.A. Ploc, M.A. Miller, J. Nucl. Mater, 64 (1977) 71.

[12] M. Yamamoto, C.T. Chan, K.M. Ho, S. Naito, Phys. Rev. B, 54 (1996) 14111.

[13] G. Jomard, A. Pasturel, Appl. Surf. Sci., 177 (2001) 230.

[14] F.-H. Wang, S.-Y. Liu, J.-X. Shang, Y.-S. Zhou, Z. Li, J. Yang, Surf. Sci, 602 (2008) 2212-2216.

[15] X. Wang, M. Khafizov, I. Szlufarska, J. Nucl. Mater, 445 (2014) 1-6.

[16] R. Yao, F.-H. Wang, Y.-S. Zhou, Acta Phys. Sinica, 58 (2009) 177-182.

[17] G. Kresse, J. Hafner, Phys. Rev. B, 47 (1993) 558.

[18] G. Kresse, J. Furthmüller, Phys. Rev. B, 54 (1996) 11169-11186.

[19] P.E. Blöchl, Phys. Rev. B, 50 (1994) 17953-17979.

[20] J.P. Perdew, K. Burke, M. Ernzerhof, Phys. Rev. lett., 77 (1996) 3865-3868.

[21] G. Jomard, T. Petit, L. Magaud, A. Pasturel, Phys. Rev. B, 60 (1999) 15624-15627.

[22] G.B. Skinner, H.L. Johnston, J. Chem. Phys., 21 (1953) 1383.

[23] G. Mills, H. Jónsson, Phys. Rev. Lett., 72 (1994) 1124-1127.

[24] G. Henkelman, B.P. Uberuaga, H. Jónsson, J. Chem. Phys., 113 (2000) 99019904.

[25] G.M. Hood, H. Zou, S. Herbert, R.J. Schultz, H. Nakajima, J.A. Jackman, J, Nucl. Mater., 210 (1994) 1-5.

[26] G. Bakradze, L.P.H. Jeurgens, E.J. Mittemeijer, Surf. Interface Anal., 42 (2010) 588-591. 


\section{${ }^{*}$ Graphical Abstracts}
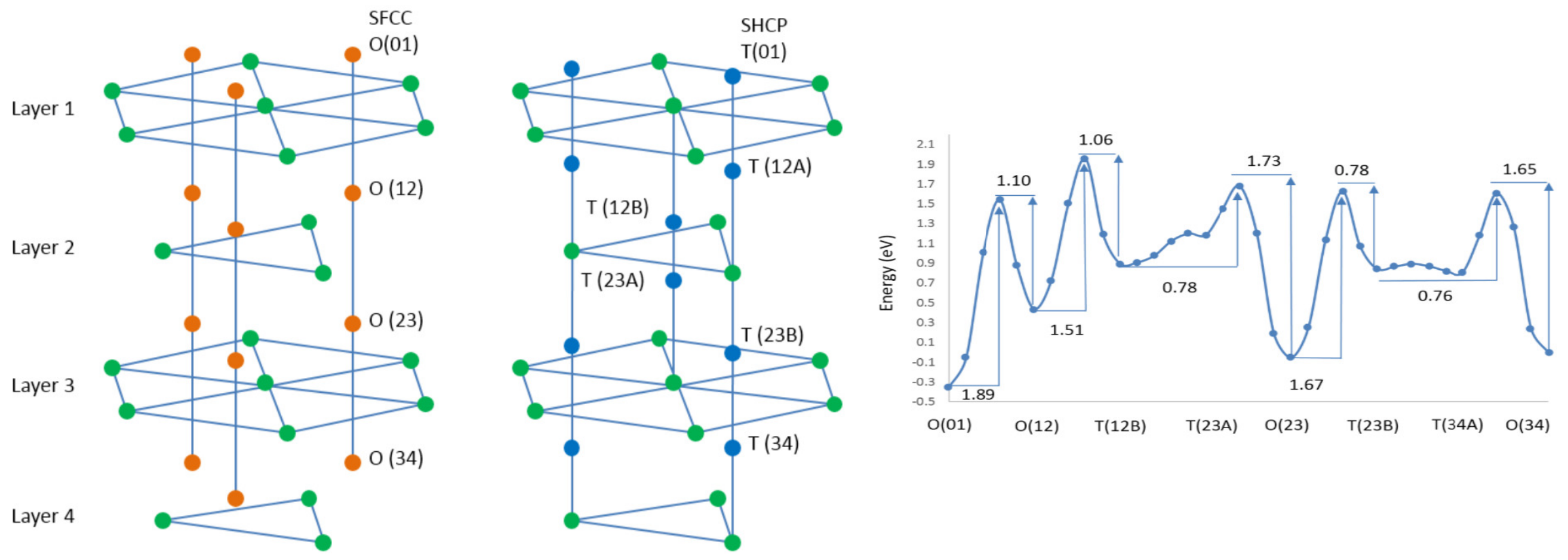